OLIVEIRA, T.E., FREITAS, D.S. e GIANEZINI, M. Perspectivas de cadeia de valor da pecuária sustentável. PUBVET, Londrina, V. 8, N. 3, Ed. 252, Art. 1665, Fevereiro, 2014.

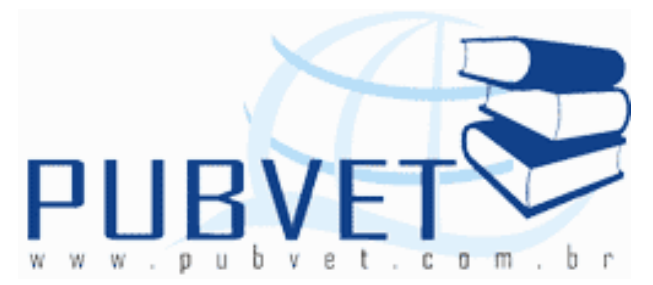

PUBVET, Publicações em Medicina Veterinária e Zootecnia.

\title{
Perspectivas de cadeia de valor da pecuária sustentável
}

Tamara Esteves de Oliveira $^{1}$, David Santos de Freitas ${ }^{2}$, Miguelangelo Gianezini ${ }^{3}$

${ }^{1}$ Mestre em Zootecnia, Doutoranda em Agronegócios na UFRGS

${ }^{2}$ Mestre em Zoologia, Professor da UFRGS

${ }^{3}$ Doutor em Agronegócios, Professor do PPGDS da UNESC

\section{Resumo}

Um dos grandes desafios que se apresenta ao agronegócio é o atendimento dos anseios dos consumidores, conciliando o aumento da produção a processos que estejam dentro das expectativas destes elos demandantes. A cadeia produtiva da carne bovina por apresentar problemas devido à ineficiência e alto impacto ambiental tem sido foco de discussões sobre produção e sustentabilidade. Portanto, entender como uma cadeia pode ser sustentável e quais são as soluções inovadoras nessa área torna-se fundamental. Para tanto, o presente estudo se propôs a conhecer as características da cadeia da carne bovina no Brasil para desenvolvimento de perspectivas de cadeia de valor da pecuária sustentável, buscando as orientações, ações e respostas dos diversos agentes envolvidos.

Palavras-chave: desenvolvimento sustentável; diferenciação de produto. 
OLIVEIRA, T.E., FREITAS, D.S. e GIANEZINI, M. Perspectivas de cadeia de valor da pecuária sustentável. PUBVET, Londrina, V. 8, N. 3, Ed. 252, Art. 1665, Fevereiro, 2014.

\section{Value chain perspectives in sustainable livestock}

\section{Abstract}

A major challenge that presents itself to agribusiness is to attend consumers' expectations, increasing food production processes that are within the precepts of sustainable development. The beef supply chain faces inefficiencies and high associated environmental damaging its image for the consumers, especially in the international market. Therefore, understanding how a supply chain can be sustainable and, what the innovative solutions available are, becomes critical. The present study aims to identify the characteristics of Brazilian beef supply chain to develop prospects for a sustainable livestock value chain, seeking guidelines, actions and responses of the stakeholders involved.

Keywords: sustainable development, product differentiation.

\section{INTRODUÇÃO}

Um dos grandes desafios que se apresenta ao agronegócio é o atendimento dos anseios dos consumidores, conciliando o aumento da produção a processos que estejam dentro das expectativas destes elos demandantes. Questões como o bem-estar animal, respeito às leis trabalhistas, segurança alimentar e proteção e preservação dos recursos naturais são relevantes para o futuro da produção de alimentos.

As discussões em relação às consequências da produção têm evoluído, passando a abranger o crescimento econômico e suas contribuições para a sociedade (SOCOLOW, 1994) e o seu impacto para os recursos naturais. Estas arguições tornaram as práticas sustentáveis de negócios um pré-requisito para fornecedores de quaisquer cadeias de fornecimento (SEURING, 2004).

De forma que a inclusão da rentabilidade como parte desse foco econômico é fundamental para a inovação e competitividade da bovinocultura brasileira, na qual grande parte da produção tem a exportação como destino, 
OLIVEIRA, T.E., FREITAS, D.S. e GIANEZINI, M. Perspectivas de cadeia de valor da pecuária sustentável. PUBVET, Londrina, V. 8, N. 3, Ed. 252, Art. 1665, Fevereiro, 2014.

estando, portanto, sujeita às expectativas do mercado internacional, no qual estas questões são emergentes.

Frente a esta perspectiva, diversos elos produtivos, envolvidos com a bovinocultura de corte no Brasil, têm buscado responder a este desafio, almejando o atendimento desta demanda. Paralelamente, a pesquisa científica tem procurado respostas e alternativas que atendam a esta evolução, buscando a resolução deste empasse de aumentar a produção para atender as demanda e as expectativas quanto à forma de produção.

Com o intuito de contribuir com essa discussão, o presente estudo se propôs a conhecer as características da cadeia da carne bovina no Brasil para desenvolvimento de perspectivas de cadeia de valor da pecuária sustentável, buscando as orientações, ações e respostas dos diversos agentes envolvidos.

\section{CADEIA DE VALOR}

A cadeia de valor analisa uma firma em suas atividades de relevância estratégica para possibilitar a compreensão do comportamento dos custos e das fontes existentes e potenciais de diferenciação em cada processo do negócio (PORTER, 1985). Dessa forma, todas as firmas devem ser consideradas como um conjunto de atividades executadas para projetar, produzir, comercializar, entregar e sustentar seu produto.

Ademais, o resultado do empreendedorismo é a criação, aprimoramento, realização e renovação de valor, não apenas para os proprietários, mas para todos os participantes e interessados (TIMMONS \& SPINELLI, 2004), voltando a ressaltar os benefícios para a comunidade.

Além disso, são sugeridas características comparáveis entre ecoempresários, empresários socialmente responsáveis ou para o empreendedorismo sustentável (COHEN \& WINN, 2007).

Frente a esta valorização, a criação de visão e direcionamento para satisfazer os desejos dos consumidores finais são diretamente relacionados a 
OLIVEIRA, T.E., FREITAS, D.S. e GIANEZINI, M. Perspectivas de cadeia de valor da pecuária sustentável. PUBVET, Londrina, V. 8, N. 3, Ed. 252, Art. 1665, Fevereiro, 2014.

soluções inovadoras para as questões ambientais e no esgotamento dos recursos não-renováveis e sistemas ecológicos (LARSON, 2000).

Nesta perspectiva, um bom empresário é aquele que entende que os consumidores, principalmente europeus e americanos, demanda produtos éticos com preocupação sobe as questões sociais e ambientais, e que deste produto deriva o seu lucro (PORTER, 1985; LUSSIER, 2006; QUAYLE, 2006).

Desenvolvimento sustentável como conceito e como prática é, portanto, promovido através de vários mecanismos do mercado, às vezes por firmas individuais e algumas vezes por atividades do setor como o GlobalGAP (MULLER et al. 2009; VERMEULEN, 2008).

\section{PECUÁRIA SUSTENTÁVEL}

Os efeitos do modelo de produção vigente desde a revolução industrial se tornaram preocupantes conforme afetaram os recursos naturais, levando à busca por alternativas de desenvolvimento e processos produtivos como foco de pesquisas, ações e políticas governamentais.

Concomitantemente, as prospecções da FAO desde 2008 sinalizam a necessidade de duplicação da produção de alimentos para atender a demanda em 2030, principalmente no que diz respeito à proteína de origem animal. Comunhão destes dois fatores exige a adaptação do processo produtivo, o qual encontra como grandes desafios aumentar a produção e diminuir o comprometimento dos recursos não renováveis necessários para esta produção (FOLEY et al. 2011).

O Brasil é considerado como a última fronteira agropecuária, reunindo terras agriculturáveis, água e desenvolvimento tecnológico (PINEDA, 2002) capaz de atender esta demanda. No que se refere ao agronegócio brasileiro, as instituições de pesquisa tem colaborado para a construção de um sistema produtivo eficiente e competitivo (EMBRAPA, 2008), mas que ainda procura equilíbrio econômico e socioambiental para manter sua competitividade. 
OLIVEIRA, T.E., FREITAS, D.S. e GIANEZINI, M. Perspectivas de cadeia de valor da pecuária sustentável. PUBVET, Londrina, V. 8, N. 3, Ed. 252, Art. 1665, Fevereiro, 2014.

O termo Desenvolvimento Sustentável foi proposto inicialmente 1987, quando a Comissão Mundial sobre o Meio Ambiente e Desenvolvimento, publicou o relatório Our Common Future, no qual o termo foi conceituado como aquele capaz de "atender às necessidades do presente sem comprometer a capacidade das gerações futuras de satisfazer suas próprias necessidades" (BRUNDTLAND, 1987).

Desde então, procuram-se alternativas ditas sustentáveis para satisfazer essas demandas da sociedade. No início do século XXI, conglomerados privados ingressaram neste cenário e agregando eficiência e competitividade como fatores de sustentabilidade econômica neste processo de desenvolvimento.

Ao longo dos últimos anos, as áreas ocupadas pela produção pecuária avançaram, sobretudo sobre as regiões Centro-Oeste e Norte do país, com crescimento de, aproximadamente, $40 \%$ do efetivo bovino. Entretanto, esta expansão ocorreu em áreas de complexidade legal e ambiental, nas quais o crescimento do rebanho foi de $178 \%$ (VERONEZ, 2009), comprometendo esta nova preocupação com a sustentabilidade.

O avanço sobre este território de preocupação e visibilidade mundiais aumentou as pressões sobre a pecuária de corte, principalmente após a publicação do Livestock's long shadow no qual essa é considerada uma das principais responsáveis pela emissão de gases do efeito estufa e degradação ambiental, envolvendo frigoríficos e redes de varejo. Contudo, com o aumento da demanda por produtos pecuários, sinalizado pela FAO, seu papel foi revisto no Review of evidence on drylands pastoral systems and climate change, em que foram apresentadas boas práticas de manejo que podem tornar a pecuária uma das atividades mais mitigadoras desses gases (NEELY, 2009).

Dentro deste contexto, a pecuária sustentável surge como iniciativa dos agentes da cadeia produtiva da carne bovina como resposta a estas exigências. Principalmente porque, independente da comprovação da responsabilidade que esta atividade tenha sobre a degradação dos recursos 
OLIVEIRA, T.E., FREITAS, D.S. e GIANEZINI, M. Perspectivas de cadeia de valor da pecuária sustentável. PUBVET, Londrina, V. 8, N. 3, Ed. 252, Art. 1665, Fevereiro, 2014.

naturais, a sustentabilidade já está difundida sobre o perfil do novo consumidor, que vai exigir cada vez mais produtos com esta comprovação.

Desta maneira, diversas firmas vêm estruturando seu planejamento para se adaptar a essas novas demandas, transformando-as em oportunidade de mercado.

$\mathrm{Na}$ pecuária de corte, diversos pesquisadores indicam técnicas de produção que aumentem a produtividade para o melhor aproveitamento das áreas já utilizadas sem comprometer outras áreas de preservação.

Além disso, o uso de tecnologias que aumentem a taxa de desfrute, tornando os sistemas mais eficientes, diminui o tempo que o animal permanece no pasto, utilizando menos recursos e possibilitando a diminuição da área utilizada (PEREZ, 2009). Dentre essas práticas, a integração com outros sistemas produtivos e o aumento da eficiência alimentar se destacam.

Esta diferenciação da pecuária na Europa (CALKER et al. 2005) indica a proteção e melhoria do ambiente natural, bem-estar animal e condições das comunidades locais, produtividade e eficiência. Nos Estados Unidos, esta estratégia caminha para a uma produção natural, em pastagens e sem promotores de crescimento, antibióticos ou esteroides, alimentados sem ingredientes de origem animal ou artificiais. A proposta brasileira melhor estruturada é a pecuária orgânica, que já certifica animais produzidos a pasto, com produtos homeopáticos e fitoterápicos (DOMINGOS, 2005).

Todos estes conceitos e proposições carecem de comprovação científica quanto aos seus resultados e impactos reais sobre os pilares sustentáveis sob os quais estão sendo construídos.

Após o estabelecimento e a harmonização destes preceitos entre os países, torna-se necessário compreender as percepções dos consumidores, produtores e dirigentes das redes de varejo para que a cadeia produtiva possa se organizar e atender a essa nova demanda. Além disso, seria necessário o esclarecimento do real impacto da pecuária sobre o ambiente, para que, a partir de dessa caracterização, possam ser propostos novos sistemas de produção. 
OLIVEIRA, T.E., FREITAS, D.S. e GIANEZINI, M. Perspectivas de cadeia de valor da pecuária sustentável. PUBVET, Londrina, V. 8, N. 3, Ed. 252, Art. 1665, Fevereiro, 2014.

No entanto, para que essa evolução seja bem-sucedida é necessário que as ferramentas gerenciais sejam apropriadas. Após esses passos, será necessária a determinação de indicadores claros e aplicáveis aos sistemas de produção de carnes para avaliar sustentabilidade, o que seria fundamental para a certificação e consequente comprovação de um sistema produtivo realmente sustentável e capaz de modificar a estrutura desta atividade.

\section{PROPOSIÇÕES}

Esta problemática apresentada sinaliza a necessidade de afastar-se da agricultura de subsistência, transformando-as em pequenas firmas, passíveis de progressão para grandes firmas ou cooperativas capazes de modificar a estrutura local, gerando empregos e contribuindo para prosperidade regional (RAS E VERMEULEN, 2009).

Como parte desse processo é fundamental que os agentes da porção final da cadeia produtiva como os consumidores e o varejo sejam considerados as bases para esse tipo de abordagem, pois normalmente as observações e preferências destes elos não são incorporadas à análise das cadeias de commodities (PELUPESSY E KEMPEN VAN, 2005). Estes agentes devem ser analisados conforme sua complexidade de forma a possibilitar um exame digno desta esfera produtiva (HUGHES, 2000).

Diversas vantagens competitivas são apresentadas para a sustentabilidade desta atividade no Brasil: disponibilidades de solo, água e luz solar; recursos humanos de baixo custo; oferta de insumos; acesso à pesquisa e consequentes tecnologias de produção; diversidade climática, adaptabilidade da raça zebuína aos trópicos e afinidade dos produtores o criador (PINEDA E ROCHA, 2002; CARVALHO E ZEN, 2010).

No entanto, estas vantagens competitivas carecem de valorização, sustentabilidade, transparência e valorização ao longo da cadeia produtiva para que possam ser convertidas em um valor real associado ao produto. $O$ processo de transformação sequencial a partir das entradas (insumos), através 
OLIVEIRA, T.E., FREITAS, D.S. e GIANEZINI, M. Perspectivas de cadeia de valor da pecuária sustentável. PUBVET, Londrina, V. 8, N. 3, Ed. 252, Art. 1665, Fevereiro, 2014.

de sua transformação até as saídas e distribuição ao consumidor final deveria dar sequencia aos processos e orientações provenientes dos outros elos produtivos, a fim de adicionar valor a cada passo de produção produtos ou serviços (HENDERSON et al. 2002).

Buscando aproximar a teoria de cadeia de valor ás propostas de sustentabilidade, este artigo busca decompor a cadeia produtiva da carne brasileira em suas propostas de atividades de relevância estratégicas (cadeia de valor) e perceber potenciais pontos de diferenciação do processo de produção para agregar valor ao produto final (Figura 1).

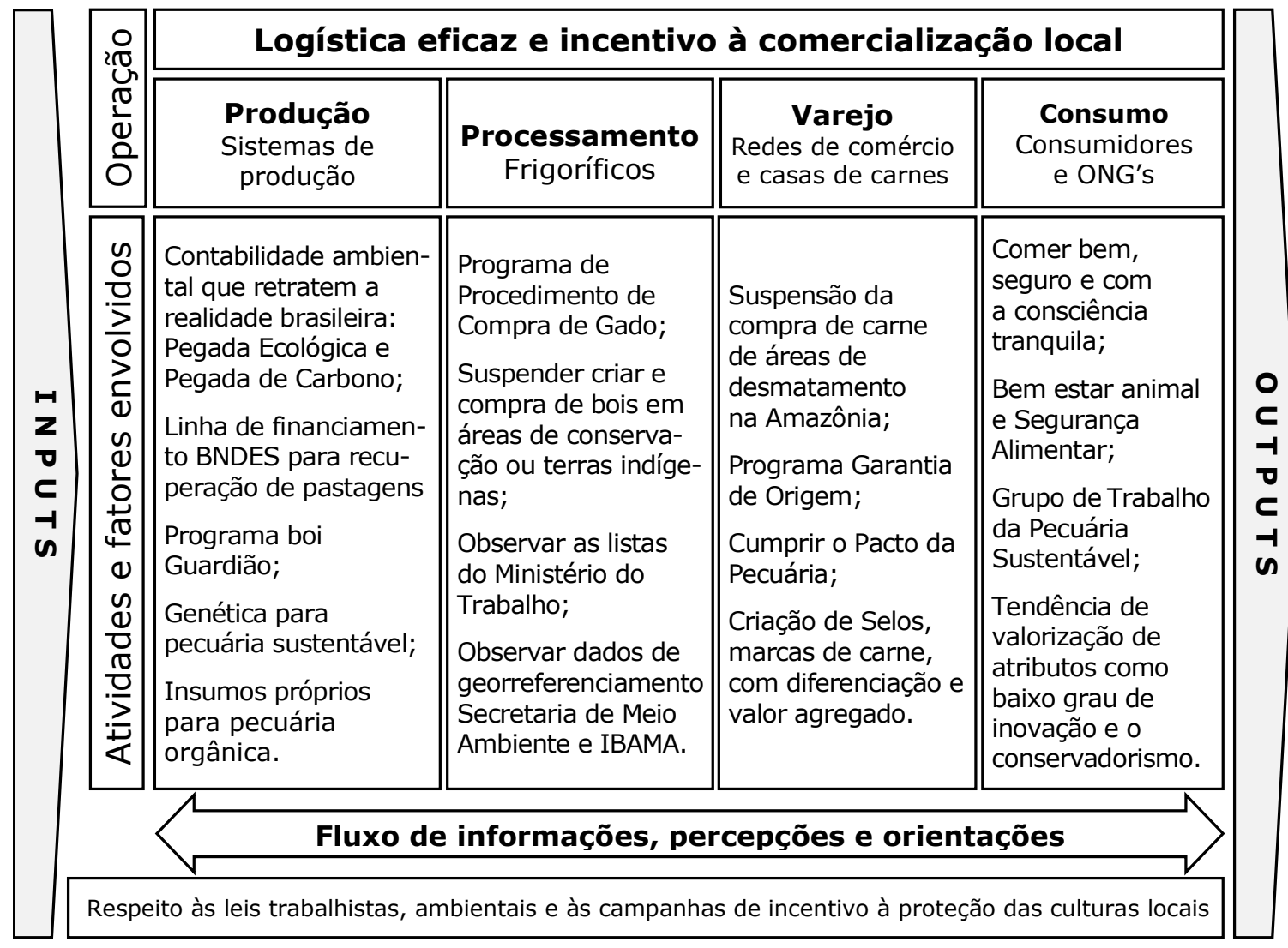

Figura 1 - Fluxograma das orientações e percepções da cadeia de valor da pecuária sustentável ${ }^{1}$ Fonte: Elaborado pelos autores.

${ }^{1}$ Elaborado a partir de informações de GIANEZINI et al., 2011; Barcellos, et al. 2010; Cerri e Carvalho, 2010; GRUPO Carrefour, 2010; Grupo JBS, 2010; GRUPO Pão de Açúcar, 2010; Rodrigues, 2010; Carfantan, 2009; Valentin e Andrade, 2009; Nascimento, 2009; IFC, 2008; PACTO, 2008; Domingos, 2005; Cavalcanti, 2004. 
OLIVEIRA, T.E., FREITAS, D.S. e GIANEZINI, M. Perspectivas de cadeia de valor da pecuária sustentável. PUBVET, Londrina, V. 8, N. 3, Ed. 252, Art. 1665, Fevereiro, 2014.

\section{CONSIDERAÇÕES FINAIS}

Diversas orientações e percepções foram destacadas através do levantamento bibliográfico realizado. No entanto, não foram identificados nos artigos científicos, arquivos do governo ou divulgação da mídia, fatores relacionados ao marketing e real comercialização com preços diferenciados destes produtos relacionados a essa nova percepção de pecuária.

O estabelecimento de uma cadeia de valor que tire proveito da tendência de valorização dos produtos ditos "conscientes" só será viável quando barreiras como a assimetria de informação e falta de coordenação hoje tão presentes na cadeia produtiva da carne bovina brasileira - forem ultrapassadas. O quadro proposto com os elementos para uma cadeia de valor da pecuária sustentável no Brasil tem o potencial de contribuir para a discussão destas questões, pois permite a integração de processos normalmente analisados em separado pela pesquisa científica (COE et al. 2008).

Dentro desta construção de uma cadeia de valor, a concepção de cadeias ou redes de produção com o um sistema de fluxos de materiais e contrapesos (TURNER et al., 1994), é uma proposição interessante e valiosa de integração, caso se alcance a interação necessária com foco ambiental entre todos os agentes da rede e esta diferenciação seja percebida, confiada e valorizada pelos demandantes da cadeia produtiva da carne bovina.

\section{REFERÊNCIAS}

BARCELLOS, M. D. et al. 2010. European consumers' acceptance of beef processing technologies: A focus group study. Innovative Food Science \& Emerging Technologies, $11,721-732,2010$.

BRUNDTLAND, G. H. Our common future: the world commission on environment and development. Oxford: Oxford University Press, 1987. Disponível em: <http://www.vliz.be/projects/indicatoren/fiches/brundtland LQ.pdf>. Acesso em: 07 ago. 2010.

CALKER, K. J. et al. Identifying and ranking attributes that determine sustainability in Dutch dairy farming. Agriculture and Human Values, Dordrecht, v. 22, n. 1, p. 53-63, 2005. 
CARVALHO T. B; ZEN S. de. Caracterização da atividade pecuária de cria nos biomas Pantanal, Amazônico e Cerrado. In: CONGRESSO DA SOCIEDADE BRASILEIRA DE ADMINISTRAÇÃO, ECONOMIA E SOCIOLOGIA RURAL, 48., 2010, Campo Grande. Anais... Campo Grande: UCDB, 2010. 1 CD-ROM.

CARFANTAN, J. Y. Sustentabilidade da pecuária: uma prioridade no varejo global. In: CONGRESSO DA PECUÁRIA SUSTENTÁVEL, 1., 2010. São Paulo: Anais... São Paulo: APPS, 2010. Disponível em: <http://www.boviplan. com.br/noticia.asp?idN=360>. Acesso em: 21 ago. 2011.

CAVALCANTI, M. da R. A busca por valor na cadeia da carne. Piracicaba: Beef Point, 2004. Disponível em: <http://www.beefpoint.com.br>. Acesso em: 22 ago. 2011.

CERRI, C. C; CARVALHO, J. L. Balanço das emissões de gases do efeito estufa da pecuária brasileira. CONGRESSO DA PECUÁRIA SUSTENTÁVEL, 1, 2010, São Paulo. Anais... São Paulo: APPS, 2010. Disponível em: <http:// www.boviplan.com.br/noticia.asp?idN=360>. Acesso em: 21 ago. 2011.

COE, N.M.; DICKEN, P.; HESS, M. Global production networks: realizing the potential. Journal of Economic Geography 8: 271-295, 2008.

COHEN, B, WINN, M.I. Market imperfections, opportunity and sustainable entrepreneurship. Journal of Business Venturing 22: 29-49, 2007.

DOMINGOS, I. T. Cenário atual da pecuária bovina de corte orgânica certificada na Bacia do Alto Paraguai (BAP) - Brasil. Brasília: WWF-Brasil, vol. 11, 2005.

EMBRAPA. Secretaria de Gestão e Estratégia. V Plano-diretor da Embrapa: 2008 - 2011 2023. Brasília: EMBRAPA, 2008. 44 p. Disponível em: <http://www.cnpt.embrapa.br/aunidade/PDE_V20081230_CIMP.pdf>. Acesso em: 19 ago. 2010.

FOLEY, J. A. RAMANKUTTY, N., BRAUMAN, K. A., Et al. Solutions for a cultivated planet. Nature, 478: 337-342, 2011.

GIANEZINI, M. et al. Sustainability in Market Orientation of Brazilian Beef Industry. In: ANNUAL IFAMA WORLD FORUM AND SYMPOSIUM - THE ROAD TO 2050: SUSTAINABILITY AS A BUSINESS OPPORTUNITY, 21., 2011, Frankfurt. Proceedings... Frankfurt. IFAMA, 2011. Disponível em: <https://www.ifama.org/events/conferences/2011/cmsdocs/2011SymposiumDocs/304_Sympo sium\%20Paper.pdf>. Acesso em: 12 jun. 2013.

GRUPO CARREFOUR. Carrefour lança carne nova de marca própria. [2011]. Disponível em: <http://www.grupocarrefour.com.br/imprensa/ releases/carrefour-lanca-carne-nova-demarca-propria>. Acesso em: 20 ago. 2011.

GRUPO JBS. Pecuária sustentável. [2011]. Disponível em: <http://www. jbs.com.br/PecuariaSustentavel.aspx>. Acesso em: 22 ago. 2011.

GRUPO PÃO DE AÇÚCAR. Produtos sustentáveis. [2011]. Disponível em: <http://www.grupopaodeacucar.com.br/responsabilidade-socioambiental/ ambiental/iniciativas-5.htm >. Acesso em: 20 ago. 2011.

HENDERSON, J., DICKEN, P., HESS, M.; et al. Global production networks and the analysis of economic development. Review of International Political Economy, 9: 4436-4464, 2002. 
HUGHES, A. 2000. Retailers, knowledge and changing commodity networks: the case of the cut flower trade. Geoforum, 3: 175-190.

IFC - International Finance Corporation. World Bank Group Issue Brief Brazil Amazon Initiative. World Bank. 2008. Disponível em: <http://www.ifc.org/ ifcext/media.nsf/AttachmentsByTitle/AM08_Brazil_Amazon/\$FILE/AM08_

Brazil_Amazon_Initiative_IssueBrief.pdf>. Acesso em: 22 ago. 2011.

LARSON, A.L. Sustainable innovation through an entrepreneurship lens. Business Strategy and the Environment 9 (5): 304-317, 2000.

LUSSIER, R.N. Management Fundamentals: Concepts, Applications, Skill Development. 3rd edition Thomas South Western: Boston, MA, 2006.

MULLER, C.; et al. Perceptions at demand side and realities at supply side: a case study of the South African Table Grape Export Industry. Sustainable Development 17 (5): 295-310, 2009.

NASCIMENTO, S. Tem boi na floresta. Globo Rural, São Paulo, n. 29, p. 14-25, 2010.

NEELY, C; BUNNING, S; WILKES, A. (Ed.). Review of evidence on drylands pastoral systems and climate change: implications and opportunities for mitigation and adaptation. Rome: Food and Agriculture Organization of the United Nations, 2009. Disponível em: <ftp://ftp.fao. org/docrep/fao/012/i1135e/i1135e00.pdf>. Acesso em: 07 ago. 2011.

PACTO da pecuária. In: SEMINÁRIO CONEXÕES SUSTENTÁVEIS, 1., 2008, São Paulo. Anais... São Paulo: Fórum Amazônia Sustentável, 2008. Disponível em: <http://www.ethos.org.br/sistemas/conexoessustentaveis/ pecuaria/pacto.asp>. Acesso em: 21 ago. 2010.

PELUPESSY, W.; VAN KEMPEN, L. 2005. The impact of increased consumer-orientation in global agri-food chains on smallholders in developing countries. Competition and Change, 9: 357-381, 2005.

PEREZ, J.R., in: Redução do impacto ambiental da pecuária bovina pelo aumento de produtividade. WORSHOP PECUÁRIA SUSTENTÁVEL, 16, 2009, São Paulo, <http://www.beefpoint.com.br/default.asp?actA=7\&areaID=15 \&secaoID=326>. Acesso em: 20 abr. 2011.

PINEDA, N. R.; ROCHA, J. C. M. C. Estratégias de marketing e alianças mercadológicas na cadeia produtiva da carne bovina. In: FIGUEIREDO, F. C. (Ed.). SIMPÓSIO DE PRODUÇÃO DE GADO DE CORTE, 3., 2002, Viçosa. Anais... Viçosa: SIMCORTE, 2002.

PORTER, M.E. Competitive Advantage: Creating and Sustaining Superior Performance. Free Press-Collier Macmillan: New York-London, 1985.

QUAYLE, M. Purchasing and Supply Chain Management Strategies and Realities. Idea Group: Hershey, 2006.

RAS, P. J.; VERMEULEN, W. J. V. Sustainable Production and the Performance of South African Entrepreneurs in a Global Supply Chain. The Case of South African Table Grape Producers. Sustainable Development. 17, 325-340, 2009.

RODRIGUES, R. Seminário destaca a genética para uma pecuária sustentável. 2010. Disponível em: <http://www.agrosoft.org.br/agropag/ 213577.htm>. Acesso em: 25 ago. 2011. 
SEURING, S. Integrated chain management and supply chain comparative analysis and illustrative cases. Journal of Cleaner Production 12 (8-10): 1059-1071, 2004.

SOCOLOW, R.H. Industrial Ecology and Global Change. Cambridge University Press: Cambridge, 1994.

TIMMONS, J.A., SPINELLI, S. New Venture Creation Entrepreneurship for the 21st Century (6th ed). McGraw-Hill: New York; 700, 2004.

TURNER, R. K., PEARCE, D., BATEMAN, I. Environmental Economics: An Elementary Introduction. Hemel Hempstead: Harvester Wheatsheaf, 1994.

VALENTIN, J. F.; ANDRADE, C. M. S. Tendências e perspectivas da pecuária bovina na Amazônia Brasileira. Amazônia: Ciência \& Desenvolvimento, Belém, v. 4, n. 8, 2009. Disponível em: <http://www.basa.com.br/bancoa mazonia2/revista/edicao_08/Versao_completa.pdf>. Acesso em: 12 dez. 2011.

VERMEULEN, W.J.V. Sustainable Global Product Chains: actors, systems and mechanisms at three levels. 14th Annual International Sustainable Development Research Conference, September 21-23, 2008.

VERONEZ, D. O conflito entre pecuária sustentável e o código florestal. In: WORKSHOP PECUÁRIA SUSTENTÁVEL BEEFPOINT, 1., 2009, São Paulo. Anais... São Paulo: Beefpoint, 2009. Disponível em: <http://www. slideshare.net/andreqcamargo/o-conflito-entre-pecuriasustentvel-e-o-cdigo-florestal-1744990>. Acesso em: 12 ago. 2011. 